\title{
Equalization of Ocular Dominance Columns Induced by an Activity-Dependent Learning Rule and the Maturation of Inhibition
}

\author{
Taro Toyoizumi and Kenneth D. Miller \\ Department of Neuroscience and Center for Theoretical Neuroscience, College of Physicians and Surgeons, Columbia University, New York, New York 10032
}

\begin{abstract}
Early in development, the cat primary visual cortex (V1) is dominated by inputs driven by the contralateral eye. The pattern then reorganizes into ocular dominance columns that are roughly equally distributed between inputs serving the two eyes. This reorganization does not occur if the eyes are kept closed. The mechanism of this equalization is unknown. It has been argued that it is unlikely to involve Hebbian activity-dependent learning rules, on the assumption that these would favor an initially dominant eye. The reorganization occurs at the onset of the critical period (CP) for monocular deprivation (MD), the period when MD can cause a shift of cortical innervation in favor of the nondeprived eye. In mice, the $\mathrm{CP}$ is opened by the maturation of cortical inhibition, which does not occur if the eyes are kept closed. Here we show how these observations can be united: under Hebbian rules of activity-dependent synaptic modification, strengthening of intracortical inhibition can lead to equalization of the two eyes' inputs. Furthermore, when the effects of homeostatic synaptic plasticity or certain other mechanisms are incorporated, activity-dependent learning can also explain how MD causes a shift toward the open eye during the $\mathrm{CP}$ despite the drive by inhibition toward equalization of the two eyes' inputs. Thus, assuming similar mechanisms underlie the onset of the $\mathrm{CP}$ in cats as in mice, this and activity-dependent learning rules can explain the interocular equalization observed in cat $\mathrm{V} 1$ and its failure to occur without visual experience.
\end{abstract}

\section{Introduction}

The question of the relative roles of genetic specification vs activity-dependent self-organization in the development of cerebral cortex remains an important and controversial one. A key system for studying these roles is the development of ocular dominance columns in primary visual cortex (V1) (Crair et al., 2001; Crowley and Katz, 2002; Huberman, 2007).

In cat V1, responses early in development are dominated by the contralateral eye (Crair et al., 1998). Both physiologically and anatomically (Crair et al., 1998, 2001), inputs from the ipsilateral eye are restricted to patches within a continuous sea of contralateral-eye inputs. Then, beginning at the end of the third postnatal week, coincident with the onset of the critical period for plasticity in response to monocular deprivation, the inputs from the two eyes become roughly equalized and segregate into alternating ocular dominance columns (Crair et al., 1998). This equalization and segregation does not occur if the animal is deprived of normal visual experience by binocular lid suture (Crair et al., 1998). Nonetheless, it was repeatedly argued (Crair et al., 1998; Crowley and Katz, 1999; Katz and Crowley, 2002) that this equal-

Received Feb. 3, 2008; revised Feb. 5, 2009; accepted April 6, 2009

This work was supported by the Japan Society for the Promotion of Science (JSPS), Grant-in-Aid 1806772 for JSPS fellows (T.T.), The Robert Leet and Clara Guthrie Patterson Trust Postdoctoral Fellowship, Bank of America, Trustee (T.T.), National Institutes of Health Grant R01 EY 11001 (K.D.M.), and Research Grant RGP63/2003 from the Human Frontiers Science Organization (K.D.M.)

Correspondence should be addressed to Kenneth D. Miller, Department of Neuroscience, Columbia University, 1051 Riverside Drive, Unit 87, New York, NY 10032. E-mail: ken@neurotheory.columbia.edu.

D01:10.1523/JNEUROSCI.0492-08.2009

Copyright $\odot 2009$ Society for Neuroscience $\quad$ 0270-6474/09/296514-12\$15.00/0 ization was unlikely to arise simply from Hebbian rules of synaptic plasticity, which are typically thought to play a major role in activity-dependent self-organization (Katz and Shatz, 1996; Miller, 1996; Swindale, 1996; Katz and Crowley, 2002; MrsicFlogel et al., 2007). This was presumably based on the intuition that, in a Hebbian competition between two inputs with similar activities, the initially dominant input could not lose innervation to the initially weak input. To our knowledge, no model has been shown to explain this activity-dependent equalization of OD columns.

In mouse V1, the onset of the critical period for monocular deprivation plasticity coincides with, and depends on, a sufficient maturation of intracortical inhibition (Hensch et al., 1998; Fagiolini and Hensch, 2000; Morales, 2002). The maturation of inhibition and the associated onset of the critical period does not occur if the eyes are not opened.

Here we show how these observations can be united: sufficiently strong inhibition can force equalization of the two eyes under a Hebbian rule. If recurrent intracortical connections are inhibition-dominated, then activation of some cortical cells by one eyes' inputs evokes net inhibition that suppresses the simultaneous activation of other cortical cells. This suppresses patterns in which a majority of cortex is driven by a single eye, leaving only those in which the two eyes equally share cortex. If cortical activation tends to occur in periodic patterns, as has been shown in developing V1 (Chiu and Weliky, 2002) and as will occur in models using a "Mexican hat" pattern of excitation and inhibition, then a periodically alternating ocular dominance pattern results. For this solution to be viable biologically, it must also be 


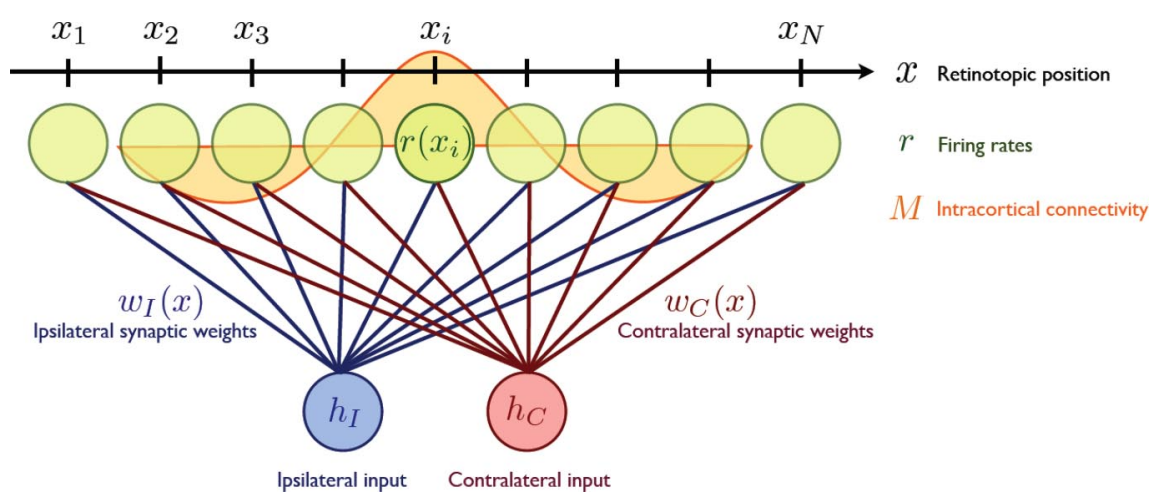

Figure 1. A schematic figure of the model. $N$ cortical neurons are lined up on a one-dimensional axis $x$. Each neuron receives feedforward input from both ipsilateral and contralateral eyes, and those synapses are modified according to activity-dependent plasticity rules. The intracortical connectivity, $M$, is a function of the distance between two cortical positions and changes its profile at the onset of the critical period when cortical inhibition matures.

the case that imbalances of the two eyes induced by monocular deprivation can lead to inequalities of the territories of the two eyes, despite the drive of inhibition to equalize these territories. We show that, in the simplest purely Hebbian models of plasticity, this solution can work but is fragile, working only in fairly restricted parameter regimes because synapses that do not drive postsynaptic neurons stay weak under Hebbian plasticity. The problem is that the simplest rules do not have robust means for synapses that have approached zero strength to regain strength after maturation of inhibition or MD. More complex plasticity rules that deal with this problem can allow both inhibitionmediated equalization of equally active eyes and monocular deprivation effects to robustly occur. To illustrate this, we study a simple modification of the model that incorporates homeostatic plasticity, which forces the average activity level of the postsynaptic cell to remain roughly constant (Turrigiano et al., 1998; Maffei et al., 2004; Turrigiano and Nelson, 2004; Mrsic-Flogel et al., 2007; Kaneko et al., 2008), as well as correlation-based plasticity, and show that under it the entire developmental sequence is more robustly replicated. An initial condition of synaptic weights consisting of ipsilateral-eye-dominated patches in a continuous sea of contralateral-eye input is stable when inhibition is weak. After maturation of inhibition, an equalized, periodically alternating ocular dominance pattern emerges, but monocular deprivation still causes an ocular dominance shift. In summary, assuming that the onset of the critical period in cats coincides with a maturation of inhibition that depends on the eyes being open, as in mice, this and activity-dependent learning rules can suffice to explain the interocular equalization observed at the onset of the critical period in cats and its failure to occur under binocular deprivation (Crair et al., 1998).

\section{Materials and Methods}

Model. We model $N=100$ pyramidal neurons in primary visual cortex receiving input from LGN (see Fig. 1). These neurons are uniformly distributed on a one-dimensional axis $x(-1<x \leq 1)$. The ends of this one-dimensional axis are connected, i.e., we use periodic boundary conditions. The model has only two inputs, one representing the contralateral eye $(C)$ and one the ipsilateral eye $(I)$. Each V1 neuron receives a connection from each of these two inputs.

We use this very simplified model because it contains the key ingredients needed to understand the problems of ocular dominance map formation, and in particular of ocular equalization and the role of inhibition in this process, while simplifying analysis and simulation-based parameter exploration. Our analysis of the 1-D system in terms of eigenmodes and constraints (supplemental material, available at www.jneurosci.org) generalizes directly to the two-dimensional case (Miller et al., 1989; Miller and MacKay, 1994; Miller, 1996; Erwin and Miller, 1998). Periodic boundary conditions simply remove boundary effects as an issue; since most of V1 is far from a boundary as measured in units of the ocular dominance spatial period, boundary conditions are unlikely to play a critical role in the processes we are studying. We consider only two input lines, whereas in reality there are many types of cells projecting to cortex (e.g., $\mathrm{ON}$ and OFF cells, and cells representing many retinotopic positions). These multiple inputs are important for studies of the organization of structured receptive fields and multifeature maps in visual cortex. However, when correlations among these inputs are such that individual receptive fields show ocular dominance segregation, then the arrangement of ocular dominance across the cortex is determined by intracortical interactions and the internal struc-

ture of receptive fields beyond the eye of dominance is irrelevant to this process (Miller et al., 1989; Miller, 1996; Erwin and Miller, 1998). Thus, a model in which inputs simply represent the two eyes is sufficient to understand the arrangement of ocular dominance across the cortex, which we are studying here.

At each time step, unrectified input firing rates $\hat{h}_{C}$ and $\hat{h}_{I}$ are drawn from a two-dimensional Gaussian distribution with mean $\left(\begin{array}{c}\nu_{C} \\ \nu_{I}\end{array}\right)$ and covariance matrix $\left(\begin{array}{ll}\nu_{C} / \tau & c / \tau \\ c / \tau & \nu_{I} / \tau\end{array}\right)$ with $\nu_{C}=\nu_{I}=10 \mathrm{~Hz}$ and $\tau=0.5 \mathrm{~s}$, where the variable $c=5 \mathrm{~Hz}$ parameterizes the strength of the covariance. The variances, of the form $\nu / \tau$, are the variances of the average rate over a time $\tau$ of a Poisson process with mean rate $\nu$. Thus, this form of covariance arises from imagining that the input firing rates averaged over a time comparable to $\tau$ are the relevant variables for the plasticity process [e.g., see Stryker (1986) and Butts et al. (2007) for evidence of long timescales in early visual system plasticity], although similar covariance structure might arise in other ways. The LGN input firing rates from the contralateral and ipsilateral eyes in that time step are then given by $h_{C}=\left[\hat{h}_{C}\right]_{+}$and $h_{I}=\left[\hat{h}_{I}\right]_{+}$. Here, $[\cdot]_{+}$is the half rectification function $[x]_{+}=x, x \geq 0$; $=0$, otherwise.

The firing rate, $r$, of a V1 neuron at position $x_{i}$ and at time step $t$ is determined by self-consistently solving (see below)

$r\left(x_{i}\right)=\left[\sum_{a=C, I} w_{a}\left(x_{i}\right) h_{a}+\sigma \xi\left(x_{i}\right)+\frac{2}{N} \sum_{j} M\left(x_{i}-x_{j}\right) r\left(x_{j}\right)-T\right]_{+}$.

Here, $w_{C}$ and $w_{I}$ are the synaptic weights of the contralateral and ipsilateral input, and $M\left(x_{i}-x_{j}\right)$ represents the strength and sign of intracortical input between the cells at positions $x_{i}$ and $x_{j}$ (described in more detail below). The noise variance is $\sigma^{2}$ and $\xi$ is a Gaussian random variable with mean 0 and variance 1 . As we explain in the Results, noise is required to induce an $\mathrm{OD}$ shift after MD given lateral interactions with strong enough inhibition to cause equalization. The noise level, $\sigma$, is varied in Results and its effects discussed. The factor $2 / \mathrm{N}$ represents the interval between adjacent neurons ( $N$ neurons over a cortex of length 2 ), and is chosen so that the total strength of intracortical input stays roughly fixed as $N$ changes. $T=1 \mathrm{~Hz}$ is a fixed threshold.

Equation 1 is solved by iteration, as follows. Let $\mathbf{r}(t)$ represent the solution for the vector of firing rates across all spatial positions at time $t$, and let $\mathbf{r}(t, n)$ represent the $n$th iteration toward the solution $\mathbf{r}(t)$. For $t=$ 0 , the first iteration, $\mathbf{r}(t, 1)$, is set to 0 ; for $t>0$, it is set to the solution of the previous time step. Then, for each $n=2,3, \ldots, \mathbf{r}(t, n-1)$ is used for the right side of Eq. 1 , and the resulting left side is $\mathbf{r}(t, \mathrm{n})$. Iterations continue until the criterion $\left|r_{i}(t, n)-r_{i}(t, n-1)\right|<10^{-3}\left([1 / N] \Sigma_{i} r_{i}(t\right.$, $n-1))$ is met for all $i$ at $n \equiv n_{c}$. At this point the solution is taken to be 
$\mathbf{r}(t)=\mathbf{r}\left(t, n_{c}\right)$. We used two parameter sets in the main text, described below: for parameter set 1 , convergence typically requires $20-50$ iterations and never $>70$; for parameter set 2 , convergence typically requires $10-20$ iterations and never $>30$. Our iteration criterion is very conservative: simply running 20 iterations without an explicit convergence criterion gave indistinguishable results with both parameter sets. The random variables - the input from the two eyes and the noise $\xi$-are fixed throughout the iterations for a given time step, but vary from time step to time step.

The strength of the intracortical connection is only a function of the difference of two neurons' cortical positions and is set to a difference of Gaussians (DOG):

$M(\Delta x)=M_{A}\left[\frac{1}{\sqrt{2 \pi \sigma_{+}^{2}}} \exp \left(-\frac{\Delta x^{2}}{2 \sigma_{+}^{2}}\right)-\frac{R}{\sqrt{2 \pi \sigma_{-}^{2}}} \exp \left(-\frac{\Delta x^{2}}{2 \sigma_{-}^{2}}\right)\right]$

with $\sigma_{+}=0.05$ and $\sigma_{-}=0.20$. Here, $M_{A}$ parameterizes the strength of recurrent versus feedforward contributions to $r$, while $R$ is the ratio of the integral of the inhibitory part of the DOG to the integral of the excitatory part of the DOG. Thus, for $R=1$, each cell receives equal strengths of total inhibitory and total excitatory input. The specific choices of $\sigma_{+}$and $\sigma_{-}$are not important to the results; all that is important is that, when inhibition is strong enough to yield equalization (see Results), the DOG select a nonzero spatial frequency whose cycle length spans a reasonable number of cells (so that effects of the discrete grid of cells do not become crucial) but spans only a fraction of the total grid (so that there can be at multiple cycles of each eye's dominance within the grid). The ranges of $M_{A}$ and $R$ that give appropriate outcomes are described in Results. For the Hebbian model of synaptic plasticity with subtractive constraints, described below, for which only a narrow range of $M_{A}$ gives appropriate outcomes, the range of $M_{A}$ that works varies as the ratio of $\sigma_{+} / \sigma_{-}$varies.

The DOG function is not meant to be a realistic model of cortical connectivity, which is three-dimensional and cell specific and develops along with the geniculocortical weights. However, it should be noted that a DOG can be achieved with short-range inhibitory connections and longer-range excitatory connections, as is observed in cortex, if feedback inhibition (the product of $E \rightarrow I$ and $I \rightarrow E$ weights) is sufficiently strong, and either $E \rightarrow E$ connections fall off more quickly with distance than $E \rightarrow I$ connections, or the two have the same distance dependence but inhibition is fast relative to excitation (the latter scenario is discussed in Kang et al., 2003; Pinto and Ermentrout, 2001).

More generally, the model requires only that the patterns of cortical activity have appropriate relative strengths of periodic activity and of spatially uniform (or low-spatial-frequency) activity, with the latter being suppressed relative to the former by inhibition. The DOG is a simple way to achieve this, but any circuitry that achieves this will yield the same model behavior. Given that ocular dominance segregation occurs and that low-spatial-frequency activity is relatively suppressed, ocular dominance will then tend to develop with a spatial period across cortex matching the spatial period of the cortical activity. Consistent with these more general ideas, periodic patterns of activity exist in V1 relatively early in development; these patterns are found in monocular as well as binocular cortex, so that they are not simply a consequence of ocular dominance segregation; and the early and weak physiological segregation of ocular dominance occurs with a spatial period that appears to match the spatial period of this activity (Chiu and Weliky, 2002).

We model the onset of the critical period (CP) as an increase in $R$. For simplicity, this increase occurs abruptly in the model, but this is not necessary for the results. During the CP, we model monocular deprivation (MD) to the contralateral eye as reductions in $\nu_{C}$ and in $c$ by a common factor $f_{\mathrm{MD}}=1 / 10$ unless otherwise stated. The between-eye correlation, $c$, is set to a nonzero value even during MD here, but setting this to zero did not change the results (data not shown). Nonzero interocular correlations during MD could arise due to residual visual signals thorough an eyelid and/or due to correlations induced by cortical feedback to LGN, as has been observed in pre-CP ferret LGN (Weliky and Katz, 1999).

We focused on two sets of parameters in this paper. The values of those parameters at different developmental phases (each phase, before $\mathrm{CP}$,
Table 1. The values of parameters before the $\mathrm{CP}$, during the $\mathrm{CP}$, and during MD

\begin{tabular}{|c|c|c|c|c|}
\hline & Parameter & & Parameter & \\
\hline & Before CP & $\mathrm{CP} / \mathrm{MD}$ & Before CP & $C P / M D$ \\
\hline$M_{A}$ & 1.1 & 1.1 & 0.8 & 0.8 \\
\hline$R$ & 0.3 & 1.2 & 0.3 & 1.0 \\
\hline$\sigma$ & $10 \mathrm{~Hz} / \tau$ & $10 \mathrm{~Hz} / \tau$ & $1 \mathrm{~Hz} / \tau$ & $1 \mathrm{~Hz} / \tau$ \\
\hline
\end{tabular}

during $\mathrm{CP}$, and during $\mathrm{MD}$, has $1.0 \times 10^{5}$ time steps) are summarized in Table 1 . The reasons for these values are established in the Results.

Learning rule. The initial condition for synaptic weights is "islands" of ipsilateral inputs in a "sea" of contralateral inputs, illustrated in Figure $2 b$. The synaptic strengths between the V1 neurons and LGN inputs are updated at each time step by one of two activity-dependent synaptic update rules. The first learning rule is a simple Hebbian learning rule with a subtractive normalization constraint, which forces the total synaptic strength received by a postsynaptic cell to remain constant (Miller et al., 1989; Miller and MacKay, 1994; Erwin and Miller, 1998). In the unconstrained Hebbian learning rule, the change $d w_{a}\left(x_{i}\right)$ in the synaptic strength $w_{a}\left(x_{i}\right)$ in one time step (where $a=C, I$ is the index describing either the contralateral or the ipsilateral eye) is described by

$$
d w_{a}\left(x_{i}\right)=\alpha h_{a}\left[r\left(x_{i}\right)-\rho \bar{r}\left(x_{i}\right)\right],
$$

where $\rho$ is a constant coefficient for the LTP/LTD threshold (see below for explanation), $\bar{r}$ is the running average of the output firing rate, updated at each time step by

$$
\Delta \bar{r}\left(x_{i}, t\right)=\beta\left(r\left(x_{i}, t\right)-\bar{r}\left(x_{i}, t-1\right)\right)
$$

with $\beta=0.02$. This means that the average is a discrete version of an exponential average across previous time steps with time constant 50 time steps. This is a long enough averaging time to obtain a reasonable (not too noisy) estimate of the average firing rates under the input ensemble for fixed weights, and short enough that it can track changes in average firing rates due to changes in weights. The learning rate is set to $\alpha=2.0 \times 10^{-5} / \mathrm{Hz}^{2}$. This number is fairly arbitrary. This rate should be small enough that learning is shaped by the average input statistics (that is, a reasonable sample of the input ensemble is seen over a time in which weight change is negligible), rather than having large changes in weights induced by individual input instantiations ("one-shot learning"). It should also be small enough that the discrete-time dynamics we use well approximates continuous-time dynamics. Both will be true if the time constant is small enough that making it smaller, while making the number of time steps proportionately larger, does not appreciably change the results. The value used is far below this threshold. Otherwise the number is simply chosen to make the outcome for a given condition (pre-CP, post-CP, post-CP-with-MD) equilibrate in the $10^{5}$ time steps we used for each condition.

One interpretation of the plasticity rule of Equation 6 is that the amount of postsynaptic activity needed to cause potentiation varies depending on the mean postsynaptic rate $\bar{r}$. However, it can also be interpreted as a statistical model of the relative rate of individual potentiation and depression events that themselves have fixed requirements for presynaptic and postsynaptic activities. Under simple models of spiketiming-dependent plasticity (STDP) (Song et al., 2000; Gerstner and Kistler, 2002) [but e.g., see critique in Lisman and Spruston (2005)], potentiation occurs when postsynaptic spike follows presynaptic spike within a fixed window, the rate of which depends on pre-post correlation (the product of the mean presynaptic and postsynaptic spike rates plus the covariance between them), while depression occurs when postsynaptic spike occurs within a fixed window before the presynaptic spike, the rate of which only depends on the product of mean presynaptic and postsynaptic spike rates. (Input temporal correlations may lead the depression rate also to have some dependence on pre-post covariance, but we neglect that here.) After averaging over the input ensemble for a given value of weights, Equation 3 says that the weight change depends on the difference between the pre-post correlation, represented in our rate model by the term $h_{a} r$, and the product of the presynaptic and postsyn- 
A
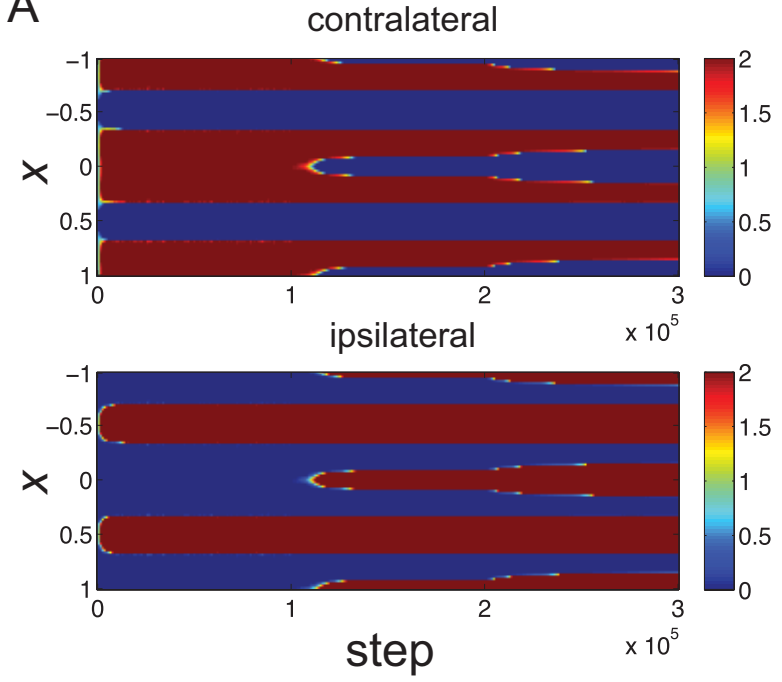

$B^{2}$

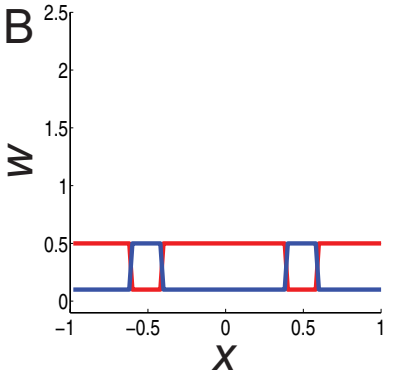

$D^{2}$

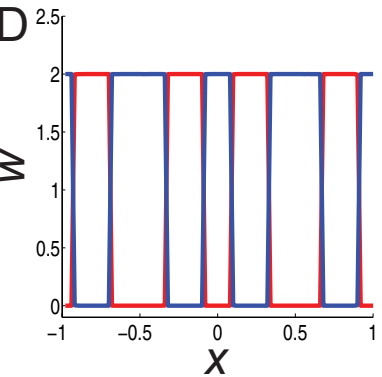

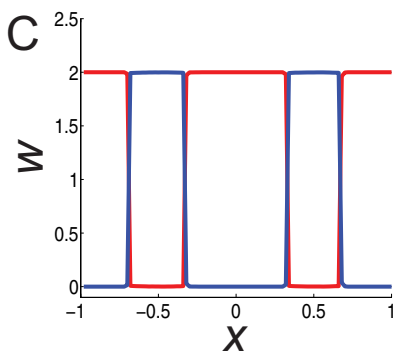

E 2.5

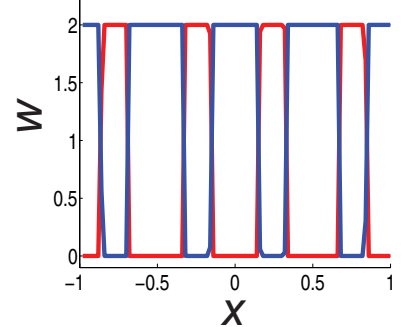

Figure 2. The evolution of synaptic weights using a Hebbian learning rule with subtractive weight normalization (Eq. 6). $\boldsymbol{A}$, The evolution of synapses carrying information from the contralateral (top) and ipsilateral (bottom) eye. Synaptic strengths are shown as color, ranging from zero (blue) to maximal (dark red). Vertical axis is position along the one-dimensional cortical grid. Horizontal axis is time: the CP starts at time 1 ( $10^{5}$ iterations), and MD of the contralateral eye is initiated at time $2\left(2 \times 10^{5}\right.$ iterations). $\boldsymbol{B}-\boldsymbol{E}$, Snapshots of the synaptic strengths from the contralateral eye (red) and from the ipsilateral eye (blue) at different times: initial state, time $0(\boldsymbol{B})$; before $(P$, time $1(\boldsymbol{C})$; during $(P$ but before $M D$, time 2 (D); and after MD, time $3(\boldsymbol{E})$. Vertical axis, Synaptic strength; horizontal axis, position along the cortical grid.

aptic mean rates, represented here by the term $h_{a} \bar{r}$. The parameter $\rho$ in Equation 6, which determines the relative size of the two terms, corresponds to the ratio of the absolute value of the integral of the negative or depressing part of the temporal function describing STDP (this function tells the weight change for a given time interval between presynaptic and postsynaptic spikes) to the integral of the positive or potentiating part [e.g., see Song et al. (2000), where the depressing part is assumed to have the larger integral].

We use $\rho=0.3$ for the simulations in the main text. This corresponds to the assumption that, when inputs and outputs are uncorrelated, potentiation strongly dominates over depression. This assumption appears to be true for plasticity based on spike bursts in early development in the LGN (Butts et al., 2007), and more generally the potentiating part of the STDP temporal function may have larger integral than the depressing part in some cortical systems [Abbott and Nelson (2000), their Fig. 2a]. As we will show, this assumption is important for monocular deprivation effects to arise along with equalization under the Hebbian learning rule with subtractive normalization. The second learning rule we will study gives a more robust solution to this problem with a more firmly established biological basis.

The normalization constraint is imposed by setting the total change $\Delta w_{a}\left(x_{i}\right)$ in the synaptic strength per time step to

$$
\Delta w_{a}\left(x_{i}\right)=d w_{a}\left(x_{i}\right)-\frac{1}{2}\left[d w_{C}\left(x_{i}\right)+d w_{I}\left(x_{i}\right)\right] .
$$

This ensures that $\Delta w_{C}\left(x_{i}\right)+\Delta w_{I}\left(x_{i}\right)=0$, and thus that the sum of ipsilateral- and contralateral-synaptic strengths at each location $x_{i}$, $w_{C}\left(x_{i}\right)+\Delta w_{I}\left(x_{i}\right)$, is constant. Equation 5 can be rewritten

$$
\Delta w_{a}\left(x_{i}\right)=\frac{\alpha}{2}\left(h_{a}-h_{b}\right)\left[r\left(x_{i}\right)-\rho \bar{r}\left(x_{i}\right)\right],
$$

where $b$ is the opposite eye to $a$. For this learning rule, synapses change their values within limits that keep synapses excitatory and bounded: the minimum and the maximum values are $w_{\min }=0$ and $w_{\max }=2$, respectively. If a change in a synaptic weight under the above rules would exceed these limits, the weight is instead set to the limit. This can cause the subtractive weight normalization constraint to be violated. The lower weight limit enforces the biological fact that geniculocortical synapses are excitatory. Without an upper weight limit, the weights can grow arbitrarily large (because weights hitting the lower limit violate the normalization constraint), so the upper limit is imposed to prevent this, but the precise value of this limit does not affect results.

Another learning rule is described by a Hebbian learning rule with a homeostatic constraint, which forces the average activity level of the postsynaptic cell to remain roughly constant (Turrigiano et al., 1998; Maffei et al., 2004; Turrigiano and Nelson, 2004; Mrsic-Flogel et al., 2007; Kaneko et al., 2008). In this case, the changes in synaptic strength are given by

$$
\Delta w_{a}\left(x_{i}\right)=\alpha\left\{h_{a}\left[r\left(x_{i}\right)-\theta\left(x_{i}\right)\right]-\gamma\left(w_{a}\left(x_{i}\right)\right)^{2}\right\}
$$

with a sliding threshold $\theta=\bar{r}^{2} / r_{0}$. The learning rate is set to $\alpha=5.0 \times$ $10^{-6} / \mathrm{Hz}^{2}$ (a quarter the size used for the previous rule to keep the rates of monocular deprivation effects approximately equal in the two rules), the reference firing rate is $r_{0}=10 \mathrm{~Hz}$, and the synaptic decay coefficient is $\gamma=10 \mathrm{~Hz}^{2}$ for $h_{a}>1 \mathrm{~Hz}$ and $\gamma=0$ otherwise. The dependency of $\gamma$ on presynaptic activity makes the learning rule consistent with observations that LTD does not occur when there is no presynaptic activity (Rittenhouse, 1999; Frenkel and Bear, 2004; Heynen, 2003). Setting $\gamma$ to a constant does not change the results of this paper except for the $f_{\mathrm{MD}} \approx 0$ behavior in Figure 7. The nonzero value of $\gamma$ sets the speed of effects of $\mathrm{MD}$ on the deprived eye and also determines the maximum synaptic weight. [After this work was completed, Linden et al. (2009) suggested a different interpretation of the monocular inactivation experiment.] Other factors are as in the previous rule.

The learning rule of Equation 7 is a combination of Hebbian plasticity and homeostatic regulation because the postsynaptic threshold separating LTP from LTD changes faster than linearly with the recent mean postsynaptic firing rate (whereas in Eq. 3, this dependence is linear). The activity-dependent threshold $\theta$ with a faster-than-linear dependence on $\bar{r}$ is a key feature of the BCM learning rule (Bienenstock et al., 1982; Cooper et al., 2004). It has the effect of causing a nonspecific increase in synaptic weights if $\bar{r} \ll r_{0}$ and a nonspecific decrease in synaptic weights if $\bar{r} \gg r_{0}$, 
thus forcing the average activity to approach $\bar{r} \approx r_{0}$. Thus, it provides a simple mathematical implementation of a homeostatic constraint. This particular, sliding-threshold form of the homeostatic rule is not critical to the results; we have obtained similar results with a rule in which the homeostatic constraint was enforced by a term separate from the LTP/ LTD term (see Discussion). The decay term $-\gamma\left(w_{a}\left(x_{i}\right)\right)^{2}$ ensures that synapses with small but nonzero presynaptic activity $\left(h_{a}>1 \mathrm{~Hz}\right)$ decay back to zero in the absence of sufficiently strong pre/post correlations. This ensures that, under monocular deprivation, the deprived-eye synapses weaken before the open-eye synapses strengthen. Again, this can alternatively be addressed by using separate homeostatic and LTP/LTD terms (see Discussion). For this learning rule we only enforced a minimum value of synaptic strength, $w_{\min }=0$. Again, if a change in a synaptic weight under the above rule would exceed this limit, the weight is instead set to $w_{\text {min }}$. We set no explicit maximum value because the weight decay term keeps synaptic weights from growing too large.

\section{Results}

We begin by describing the basic intuition and mathematics behind our results, then demonstrate the results in simulations.

To understand how sufficiently strong inhibition can force equalization, we rely on the following basic intuition about Hebbian rules (Miller, 1990, 1996). If activation of cortical location A tends to cause excitation of cortical location $\mathrm{B}$, then inputs to $\mathrm{A}$ tend to promote development of coactive inputs to $\mathrm{B}$. This is because the inputs to A tend to activate the A cells, hence to cause excitation at $\mathrm{B}$, and hence to assist coactive inputs at $\mathrm{B}$ in becoming strengthened by a Hebbian mechanism. Conversely, if activation of cortical location A tends to cause inhibition of cortical location $\mathrm{B}$, then inputs to $\mathrm{A}$ tend to suppress development of coactive inputs to B. Now suppose recurrently driven inhibition becomes strong enough that the integrated effect on any one cortical location of activating all of cortex is inhibition. Assuming interactions are sufficiently localized, this means that a large enough region dominated by one eye will tend to suppress development of that eye's inputs at the center of the region, which in most competitive scenarios will lead to development of the other eye's inputs there. Thus, any large region dominated by one eye must break up and allow emergence of the other eye's inputs.

Mathematically, this can be seen from the analysis of simple models of ocular dominance development involving a Hebbian learning rule and conservation of total postsynaptic strength (Miller et al., 1989; Miller, 1990; Erwin and Miller, 1998) [see also Swindale (1980), their Appendix, and supplemental material, available at www.jneurosci.org]. It was shown that, if input correlations are such as to lead to the development of ocular dominance segregation, the overall period of this segregation is determined by an intracortical interaction function $K(x)$ describing the influence of activity at one cortical location on activity at another location a distance $x$ away. In particular, the growth of a periodic pattern of ocular dominance with period $2 \pi / k$ occurs at a rate $\tilde{K}(k)=(1-\tilde{M}(k))^{-1}$, where $M(x)$ describes the strength and sign of connection between two neurons separated by distance $|x|$ and $\tilde{M}(k)$ is the Fourier transform of $M(x)$ at frequency $k$. In this picture, all patterns with nonzero $k$ involve a periodic oscillation between the eyes (the "AC" patterns) and thus involve overall equality of the two eyes. Inequality can arise only if the pattern with $k=0$ (the "DC" pattern-the pattern in which one eye dominates everywhere) can grow. The growth rate of the DC pattern is $\tilde{K}(0)=(1-\tilde{M}(0))^{-1}$, where $\tilde{M}(0)$ is proportional to the integral over space of the intracortical connectivity: $\tilde{M}(0) \propto$ $\int d x M(x)$. Thus, for dominant inhibition, $\tilde{M}(0)<0$, and as the dominance of inhibition increases, $\tilde{M}(0)$ becomes increasingly negative and the corresponding growth rate $\tilde{K}(0)$ becomes in- creasingly small. If the growth rate of the DC pattern $\tilde{K}(0)$ is sufficiently small relative to those of the fastest-growing AC patterns $\tilde{K}(k)$ with $k \neq 0$, then, given reasonable nonlinearities (for example, limiting the synaptic strengths to remain positive and smaller than some maximum), the pattern with $k=0$ will be suppressed in favor of patterns with nonzero $k$. This causes any inequality between the eyes to be eliminated (for further analysis, see supplemental material, available at www.jneurosci.org). The basic intuition behind this analysis - that if a given location receives strong enough net inhibition from all other locations, this prevents overall domination by a single eye-is likely to be robust across a variety of proposed ocular dominance models.

An additional requirement for a realistic model is that, although the strong inhibition enforces overall equality of the two eyes when they have equal activities, it still allows the development of inequality in response to monocular deprivation (MD). Let us refer to the two eyes as the open and the closed eye, even before MD is initiated. Once inhibition matures and the two eyes equalize, activation of the open eye elicits net inhibition in columns dominated by the closed eye. This is due both to inhibition from columns dominated by the open eye and to the fact that open-eye inputs to the closed-eye columns have become very weak. After MD is initiated, it remains true that activation of the open eye leads to inhibition of cells that were dominated by the closed eye, even if MD leads to loss of strength of the closed-eye inputs. Thus, for an OD shift to occur under MD, something must strengthen open-eye inputs to closed-eye columns even when they cannot drive the postsynaptic cell, as has been observed in experiments (Mioche and Singer, 1989). These inputs must be strengthened to the point that they can drive the cell when the open eye is activated despite the inhibition from openeye-dominated columns. We show that noise in postsynaptic firing can contribute to this, and more generally, homeostatic plasticity can accomplish this. Since this work was largely completed, it has indeed been shown that homeostatic plasticity is the mechanism causing strengthening of the open eye after MD (Kaneko et al., 2008). (Homeostatic weakening of inhibition onto these cells could be part of this process, but for simplicity we do not model inhibitory plasticity here.)

We demonstrate the effectiveness of these ideas in simulations of a one-dimensional grid of cortical cells each receiving two inputs, one representing each eye, where the weights of the input synapses develop under simple learning rules (see Fig. 1). We model $N=100$ excitatory layer 4 neurons in primary visual cortex, which are uniformly distributed on a one-dimensional axis $x$ $(-1<x \leq 1)$, where each neuron is labeled by its cortical position $x$. Each V1 neuron receives input from both the contralateral $(C)$ and the ipsilateral ( $I$ ) eye, from other V1 neurons through intracortical connections, and additional noisy input modeled as Gaussian random noise with mean 0 and variance $\sigma^{2}$. For simplicity we consider the feedforward connections from a given eye to a cortical cell to be described by one effective input, which has been shown to be adequate to understand the development of patterning across cortex of ocular dominance when input correlations are appropriate to yield ocular dominance segregation (Miller et al., 1989; Miller, 1990, 1996). These feedforward synaptic strengths from the contralateral and ipsilateral eyes to a neuron at position $x$ are described by $w_{C}(x)$ and $w_{I}(x)$, respectively, and they are subject to activity-dependent plasticity (see Materials and Methods). The strength of the intracortical connection between two neurons is modeled as a function of the difference of their positions and is set to a difference of Gaussians (DOG). As discussed in Materials and Methods, the key require- 
A
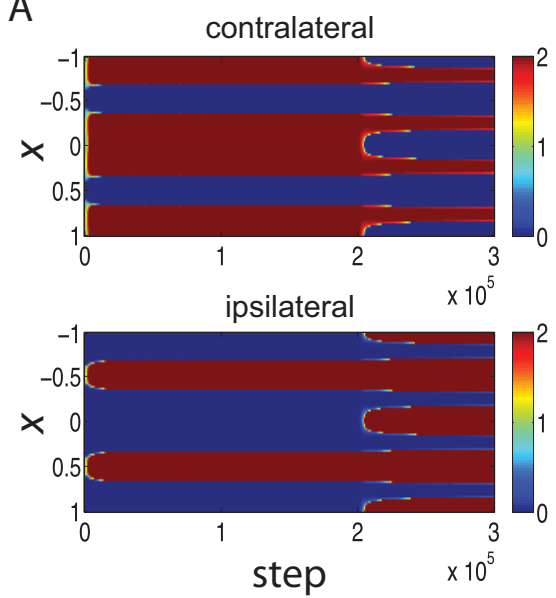

C
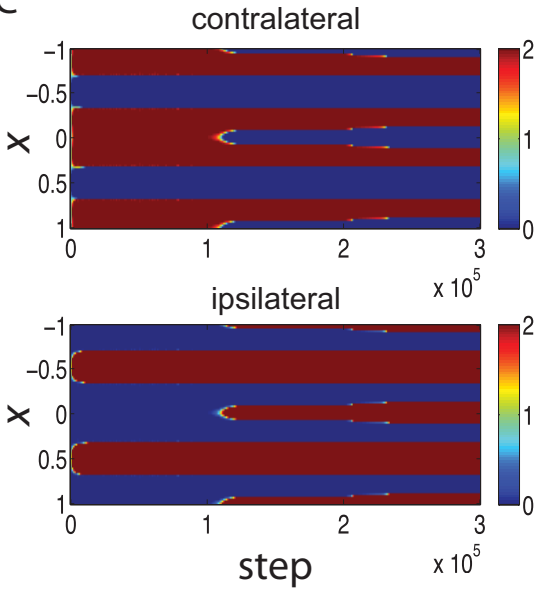

B
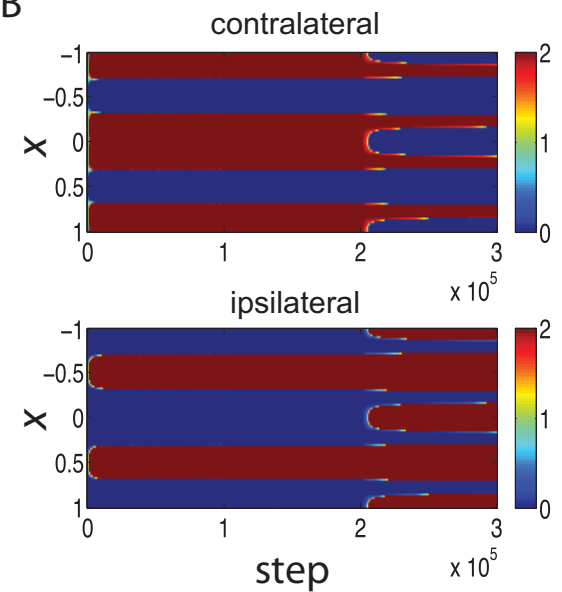

D
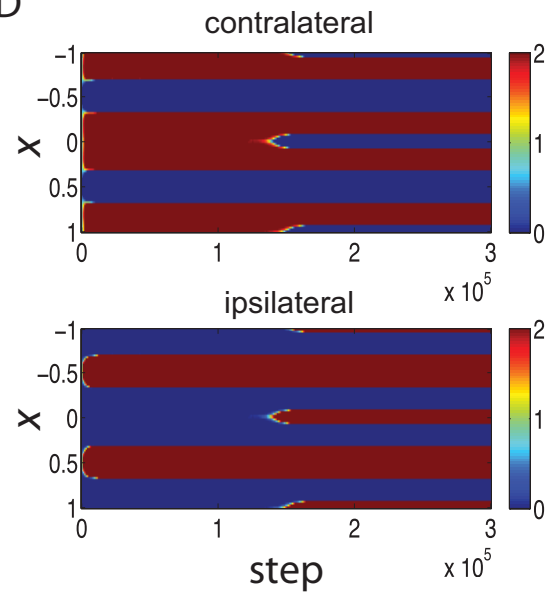

Figure 3. Parameter dependence of the development of $O D$ columns using a Hebbian learning rule with subtractive weight normalization (Eq. 6). Panels plot synaptic strengths in color as a function of cortical position, $x$, and simulated time steps as in Figure $2 A$. Explicitly mentioned parameters are changed, whereas the other parameters are as in the parameter set 1 . The learning rule of Equation 6 is sensitive to parameter choices: with intracortical connections that are too weak, $M_{A}=1.0$, the two eyes do not equalize even after the maturation of inhibition $(\boldsymbol{A})$; with inhibition during the $C P$ that is too weak, $R=1.0$, the two eyes do not equalize $(\boldsymbol{B})$; with too little noise, $\sigma^{2}=3 \mathrm{~Hz} / \tau$, almost no OD shift is observed during the simulated MD (C). $\boldsymbol{D}$, For balanced LTP and LTD, $\rho=1.0$, no OD shift is observed after MD.

ment is not that cortical circuitry be described by a DOG, but that the cortical circuitry produce periodic patterns of activity, as has been observed (Chiueliky and Weliky, 2002), with the balance between periodic and spatially uniform patterns of activity varying appropriately with inhibition. The DOG is a simple circuitry that achieves this. Two important parameters characterize this DOG: $M_{A}$ represents the strength of recurrent versus feedforward contributions to postsynaptic activities, whereas $R$ is the ratio of the integral of the inhibitory part of the DOG to the integral of the excitatory part of the DOG. We model the onset of the critical period (CP) as a threefold to fourfold increase of cortical inhibition (Morales et al., 2002). Hence, $R$ changes from an excitationdominated profile $(R<1)$ to a balanced or inhibition-dominated one $(R \geq 1)$.

\section{Hebbian learning rule with a subtractive normalization constraint}

We first simulate development under a Hebbian learning rule with subtractive normalization of the total postsynaptic weight on a cell (Fig. 2), using the parameter set 1 (see Materials and Methods). The modification of synapses at each time step depends on the covariance between the postsynaptic and presynap- tic firing rates, subject to a constraint that the total synaptic strength to each cell is held constant, which forces a competition between the eyes (Miller et al., 1989; Miller, 1990; Erwin and Miller, 1998) (see Materials and Methods). Each synapse is potentiated or depressed until it saturates to the upper or lower bound of synaptic strength $\left(w_{\max }=2\right.$ and $w_{\min }=0$, respectively). We take as our initial condition the strong bias observed in pre-critical-period cats (Crair et al., 1998): ipsilateral "islands" in a "sea" of contralateral inputs (Fig. 2B). Development before the critical period, modeled as a period in which intracortical inhibition is relatively weak (the integral of intracortical inhibition is $3 / 10$ of that of intracortical excitation, that is, $R=0.3$ ), leads synapses to go to the maximum or minimum allowed values with no change in the overall, contralateral-dominated pattern (Fig. $2 C)$. Initiation of the critical period, modeled as an increase of the strength of intracortical inhibition (the integral of intracortical inhibition becomes 1.2 times that of intracortical excitation, $R=1.2$ ), slowly leads to an equalization of the two eyes (Fig. 2D). Although inhibition drives the two eyes to have equal innervation, monocular deprivation (MD) of the contralateral eye causes a shift in favor of the open, ipsilateral eye (Fig. 2E). Here, MD of the contralateral eye is modeled as a reduction in its activity (mean, variance, and covariance with the open eye) by a factor $f_{\mathrm{MD}}=$ 1/10 (see Materials and Methods).

The outcomes under this rule are sensitive to parameter choices in at least two ways. First, they are very sensitive to the parameters describing intracortical interactions. If $M_{A}$, the strength of recurrent connections relative to feedforward, is too weak after the $\mathrm{CP}$ ( such as $M_{A}=1.0$; we used $M_{A}=1.1$ in Fig. 2), then equalization of the eyes does not occur after the onset of the CP (Fig. $3 A$ ). Much stronger inhibition during the $C P$ is required in this case for the equalization of OD columns $(R=1.8$ equalizes but $R=1.6$ does not: data not shown). On the other hand, if $M_{A}$ is too strong (such as $M_{A}=$ $1.2)$, then given weak initial inhibition $(R=0.3)$, the system becomes unstable. Strong $M_{A}$ together with stronger inhibition at the beginning to prevent instability (e.g., $M_{A}=1.2$ and $R=0.5$ during pre-CP) tends to equalize the OD columns from the beginning of the simulation even before further maturation of inhibition (data not shown). Only a narrow band of $M_{A}$ yields ocular dominance equalization only upon the maturation of inhibition. Moreover, with this model and these parameters, the inhibitory strength does not merely need to equal excitation in the intracortical connections, but actually needs to exceed it (we use $R=1.2$ ); $R=1.0$, which represents equality of total excitatory and total inhibitory strength, does not lead to equalization (see Fig. 3B).

Note that equalization can occur even with relatively weak inhibition when recurrence is strong enough that the system is 
close to instability, for the following reason. With weak inhibition, the fastest-growing pattern is an "AC" pattern with period $2 \pi / k_{\max }$ for $k_{\max } \neq 0$, but the Fourier transform of the intracortical connection function at this frequency, $\tilde{M}\left(k_{\max }\right)$, is only slightly larger than that of the "DC" pattern, $\tilde{M}(0)$. With weaker recurrence, this difference is not sufficient to cause equalization. However, moving the system close to instability means that $\tilde{M}\left(k_{\max }\right)$ becomes close to 1 so that the largest growth rate (1 $\left.\tilde{M}\left(k_{\max }\right)\right)^{-1}$ becomes both arbitrarily large and arbitrarily larger than the growth rate $(1-\tilde{M}(0))^{-1}$ of the DC mode, leading to equalization.

Second, for MD to lead to an OD shift despite inhibition strong enough to cause equalization, active synapses need to have a strong advantage over less active synapses. To achieve this (Fig. $2 E$ ), we needed to use a learning rule in which LTP strongly dominates over LTD ( $\rho=0.3$ : see Materials and Methods) along with noise in postsynaptic neuronal response. Without these two elements, equality of the two eyes is maintained even after one eye's activity is strongly reduced (see Fig. 3). This is because, during $\mathrm{MD}$, neurons in the patches dominated by the closed eye receive more lateral inhibition than excitation as a result of open eye input. Therefore, the activity levels of these neurons during open-eye input are lower than their threshold level, leading to depression of the open-eye synapses onto them. This prevents any MD shift toward the open eye. Larger noise along with a nonlinear input-output relation enables those neurons to be sometimes active in the presence of lateral inhibition. If LTP dominates over LTD, even random presynaptic and postsynaptic activity leads more active inputs to show relatively greater potentiation than less active inputs. When this is combined with a constraint preserving total synaptic strength, the result is that relatively more active synapses are potentiated while less active synapses are depressed. This allows MD shifts to occur (Fig. 2E).

These considerations explain why we used parameter set 1 (see Materials and Methods) for this model, which included high noise and a large critical period $R$ and $M_{A}$. Using parameter set 2 (see Materials and Methods) with this model does not yield equalization of the two eyes (see supplemental material, available at www.jneurosci.org).

The biological relevance of the dominance of LTP over LTD assumed here is certainly questionable, but some systems have temporal windows for spike-timing-dependent plasticity (STDP) in which integrated potentiation appears larger than integrated depression [Abbott and Nelson (2000), their Fig. $2 a$; Butts et al. (2007)]. More generally, there must be some additional mechanism that leads open-eye synapses to strengthen during MD even when they cannot drive the postsynaptic cell, as observed in experiments (Mioche and Singer, 1989). A more plausible mechanism, and one that in fact seems to drive potentiation of the open-eye during MD (Kaneko et al., 2008), is homeostatic plasticity, to which we now turn.

\section{Hebbian learning with a homeostatic constraint and weight decay}

We reasoned that the fragility of the result presented in the last section is likely to be induced by the simplicity of the learning rule studied, and that more complex biological learning rules may be more robust. One simple mechanism, though probably not the only one, that can be added to a Hebbian rule to support such robustness is activity homeostasis (Turrigiano et al., 1998; Maffei et al., 2004; Turrigiano and Nelson, 2004; Mrsic-Flogel et al., 2007; Kaneko et al., 2008): the requirement that cortical cells adjust their weights to maintain roughly constant overall levels of average activity. Both maturation of inhibition and MD will induce a reduction in the activity level of either all cells or closedeye columns, respectively, and the homeostasis will oppose this by potentiating unsaturated synapses. This strengthens the initially weak ipsilateral-eye synapses and the weak open-eye synapses in closed-eye columns, allowing them to more robustly recover from their weakness and compete. Thus, given homeostasis, even relatively weak cortical connections that are sufficiently inhibition-dominated can equalize the two eyes' innervations in cortex, and a pattern in which the innervations of the two eyes are equal can be destabilized by monocular deprivation even with low noise, as we now show.

In Figure 4 we show the same set of simulations as in Figure 2 but now using a learning rule (Eq. 7) with a homeostatic constraint rather than a weight-conservation constraint. The homeostatic constraint keeps the mean activity level of each postsynaptic cell roughly constant. For fixed input activities, this behaves similarly to the weight-conservation constraint, but if input activities are decreased by maturation of inhibition or by MD, the homeostatic constraint increases the total weight received by the cell to bring the postsynaptic activity back to the set point. As an implementation of a homeostatic rule, we used an LTP/LTD threshold that slides faster than linearly with the mean postsynaptic activity, as in the BCM learning rule (see Materials and Methods), but this particular implementation is not critical to the results (see Discussion). We also add a weight-decay term to the learning rule for inputs with nonzero activity, which causes synaptic weights of these inputs to decay in the absence of Hebbian or homeostatic strengthening. This term causes closed-eye synapses to shrink after MD before open-eye synapses grow, as observed experimentally (Mioche and Singer, 1989; Frenkel and Bear, 2004; MrsicFlogel et al., 2007; Kaneko et al., 2008). This rule is far more robust than the previous, so we now use parameter set 2 (see Materials and Methods), in which noise levels are low, total excitatory and inhibitory strength are equal $(R=1)$ during the critical period, and the parameter controlling the overall strength of recurrent connections, $M_{A}$, is slightly smaller than before, for reasons described below. This learning rule also works with parameter set 1 (see supplemental material, available at www.jneurosci.org).

The simulation proceeds much as in the previous case (Fig. 4). Before the critical period, the initial condition of ipsilateral islands in a contralateral sea (Fig. $4 B$ ) is stable (Fig. 4C). Initiation of the $\mathrm{CP}$, modeled as a strengthening of inhibition, leads to the equalization of contralateral- and ipsilateral-eye columns (Fig. 4D). Application of MD leads to the expansion of the open-eye territory and the gradual shrinking of the closed-eye territory (Fig. 4E).

With this learning rule, the equalization of ocular dominance columns occurs more robustly. Fine tuning of the strength of the intracortical connections, $M_{A}$, is not required (Fig. 5A), and inhibition that is balanced with excitation $(R=1.0$, used in Fig. 4$)$ or even somewhat weaker than excitation (Fig. $5 B$ ) is now sufficient to drive equalization. It has been suggested that cortex shows a rough balance of excitation and inhibition (van Vreeswijk and Sompolinsky, 1998; Shu et al., 2003; Wehr and Zador, 2003; Haider et al., 2006; Higley and Contreras, 2006). We use an approximately threefold increase of inhibitory strength at the onset of the critical period, consistent with the literature (Morales et al., 2002).

The difference in robustness is due to the different constraints imposed on the two learning rules. The subtractive constraint always forces the sum of contralateral- and ipsilateral-synapses to be constant at each neuron (except when synapses take the max- 


\section{B}

A
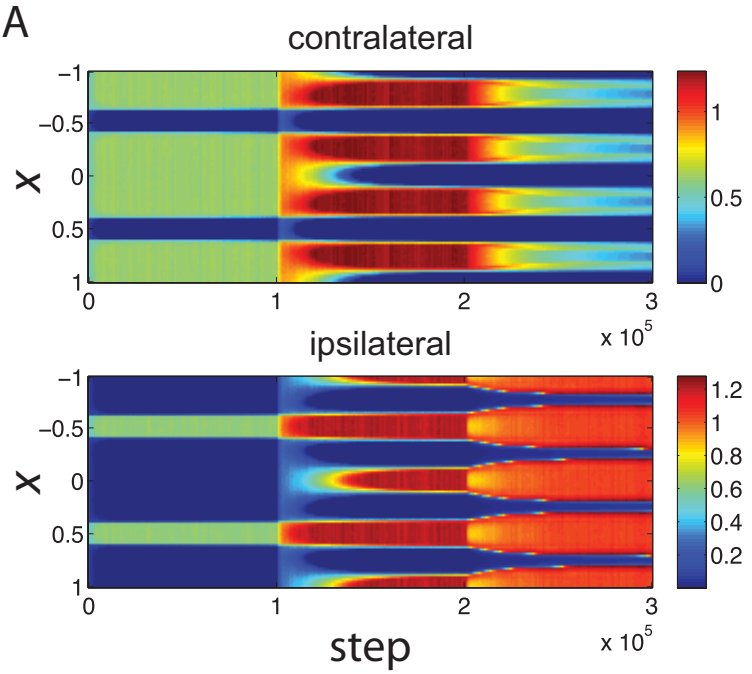

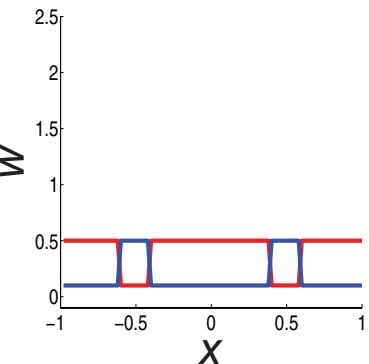

D

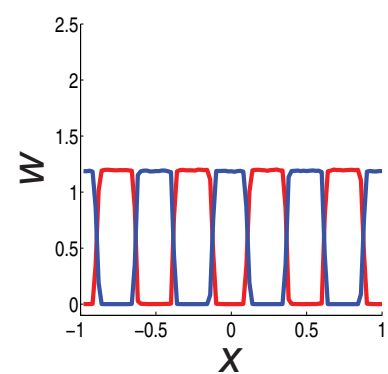

C

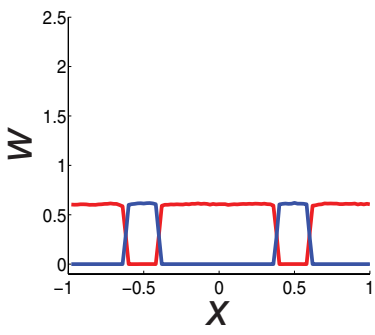

$\mathrm{E}$

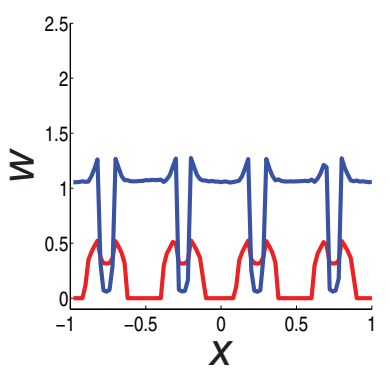

Figure 4. The evolution of synaptic weights using a combination of Hebbian and homeostatic learning (Eq. 7); conventions as in Figure 2. A, The evolution of synapses carrying information from the contralateral (top) and ipsilateral (bottom) eye, shown as color, versus time (horizontal) and position (vertical). $\boldsymbol{B}-\boldsymbol{E}$, Snapshots of synaptic strengths from the contralateral eye (red) and from the ipsilateral eye (blue) at the initial state $(\boldsymbol{B})$, before the $(P(\boldsymbol{C})$, during the $(\mathrm{P}$ but before $\mathrm{MD}(\boldsymbol{D})$, and after MD $(\boldsymbol{E})$.

A
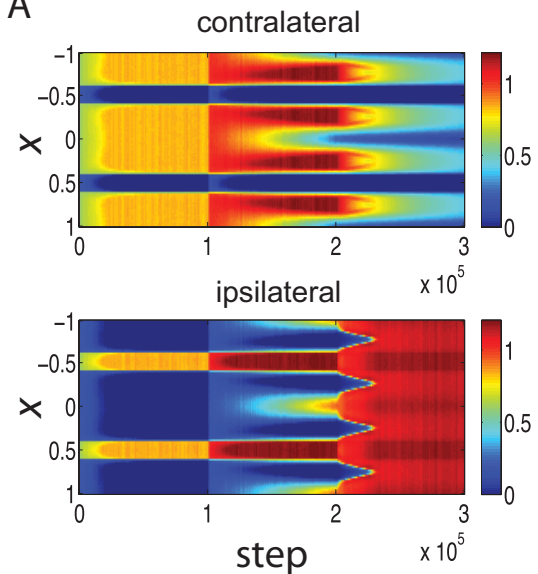

B
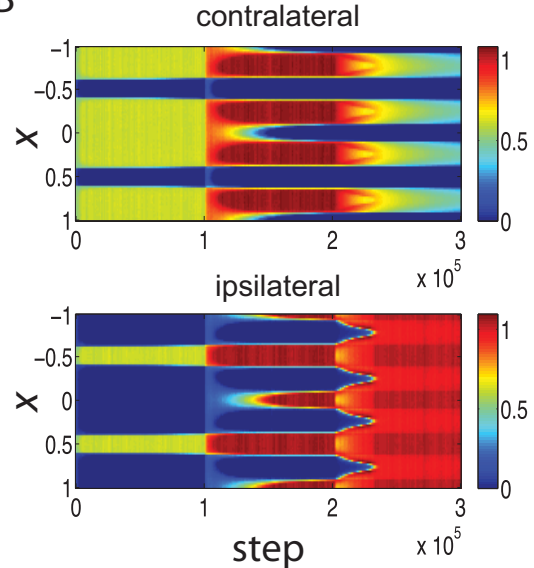

Figure 5. Parameter dependence of the development of $O D$ columns under the Hebbian-plus-homeostatic plasticity rule. Panels plot synaptic strengths in color as a function of cortical position, $x$, and simulated steps as in Figure $2 \mathrm{~A}$ or Figure $4 \mathrm{~A}$. Parameters are from parameter set 2 except where other values are explicitly noted. The homeostatic plasticity rule is more robust to parameter choices: the two eyes equalize after the maturation of inhibition with weak intracortical interactions $(\boldsymbol{A})\left(M_{A}=0.5\right)$ or with relatively weak inhibition $(B)(R=0.8)$.

imum or minimum value), so where the ipsilateral eye is initially weak, it can be difficult for it to recover under Hebbian plasticity. In contrast, the homeostatic constraint lets the summed synaptic strength increase to compensate for the reduction in cortical activity caused by the sudden maturation of inhibition, so that the ipsilateral eye can gain a foothold everywhere. The correlationbased competition can then more robustly select the pattern of innervation in which contralateral and ipsilateral synaptic strengths alternate across cortex.

With this learning rule, even a small amount of noise is enough to see the MD effect (the simulations of Fig. 4 use 1/10 the noise of those of Fig. 2). In the absence of strong input from LGN, the closed-eye synapses shrink after MD because the first term of
Eq. 7 becomes small for these less active synapses relative to the second, decay term. The open-eye synapses also shrink by a small amount due to the loss of between-eye correlation after MD but are mostly unchanged because of the intact input from the open-eye. On a slower timescale, the homeostatic learning rule along with a small amount of noise leads the open-eye synapses to grow. The homeostatic rule drives the mean postsynaptic activity, $\bar{r}$, estimated as an exponentially weighted average of past rates with a time constant of 50 iterations, to be comparable to some set point activity level $r_{0}$. After the closure of the contralateral eye, a patch of cortex that was previously driven by the closed-eye mainly receives lateral inhibition from the surrounding cortical area and therefore its LTP/LTD threshold, $\theta \sim$ $\bar{r}^{2}$, is close to zero. In the presence of small noise, those neurons near the border between the two eyes' columns will be active occasionally because they receive relatively weaker inhibition than at the center of the closed-eye dominated column. Hence, open-eye synapses at the border are slowly potentiated at a rate proportional to the activation of those neurons with coincident open-eye input until the output firing rate again approaches $r_{0}$ (Fig. $4 E$ ). On the other hand, around the center of the closed-eye dominated column, open-eye synapses cannot grow because lateral inhibition from the surrounding columns is much stronger than the noise level. The growth rate of open-eye synapses depends on the noise level, the threshold of the input-output nonlinearity and the strength of inhibition during the CP. Smaller noise, larger threshold or stronger lateral inhibition reduces the 
firing rates of neurons in previously closed-eye-dominated patches and hence slows the growth of open-eye synapses there (compare Figs. $4 A, 5 B$ ).

In the simulations presented thus far, the equalization of the two eyes is achieved by adding columns for the ipsilateral eye, so that the overall spatial wavelength of the OD columns is reduced. This issue has not been studied quantitatively, and maps were not followed across time in single animals in Crair et al. (1998, 2001). However, the data in those papers are suggestive that the wavelength does not greatly change. This can be achieved in the model for appropriate parameters. To show this, we first start the simulation from a contralaterally dominated initial condition with a smaller wavelength than previously. When inhibition matures, the two eyes are equalized, as in previous simulations, but the wavelength of OD columns is not altered (Fig. 6A). Starting from an even smaller wavelength without altering the intracortical connections, the equalized OD columns still preserve the wavelength of the initial condition (Fig. 6B). In general, the final spatial wavelength of the OD columns is determined both by the amplitude of each wavelength in the initial condition and the growth rates of the different wavelengths (as noted previously, the growth rates are proportional to $(1-\tilde{M}(k))^{-1}$, where the wavelength is $2 \pi / k$ and $\tilde{M}(k)$ is the Fourier transform at that wavelength of the function describing intracortical connections). The wavelength with the fastest growth rate will tend to dominate, but if other wavelengths have a strong advantage in the initial condition and are not too disadvantaged in terms of growth rate, they may dominate instead (Fig. 6C). Weakening the intracortical connections, as we have done in parameter set 2 by decreasing $M_{A}$ from 1.1 to 0.8 , lessens the differences between growth rates, and so allows bias in the initial condition toward a given wavelength to control the final outcome over a wider range of wavelengths. Thus, for different parameters, the final wavelength may be determined either by the intracortical connections or by the initial condition (or both). Regardless of the choice of parameters controlling this feature, however, it seems to be a robust phenomenon that maturation of inhibition equalizes the two eyes' innervations in cortex.

The MD effect in this scenario depends on the degree of weakening of the closed-eye activity. To investigate this effect, we systematically varied the strength of the closed-eye activity (mean, variance, and covariance with the open-eye) by a factor $f_{\mathrm{MD}}$ that was varied from zero to one. Figure 7 shows the mean strength of both the closed-eye and open-eye synapses after MD. If $f_{\mathrm{MD}}$ is smaller than 0.6 , we see a decrease of closed-eye synaptic
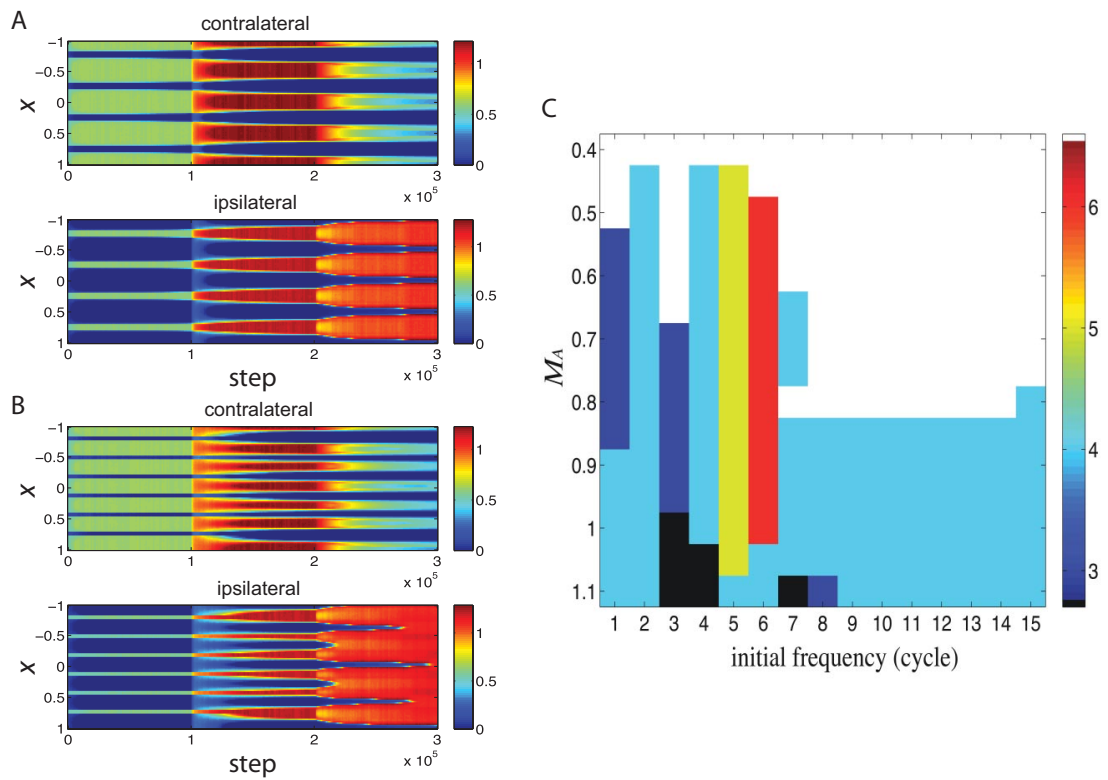

Figure 6. The spacing of ocular dominance columns is determined also by the initial condition: the evolution of synaptic weights with the Hebbian-plus-homeostatic learning rule of Equation 7, starting from an intermediate (4 cycles; $\boldsymbol{A}$ ) or high (6 cycles; $\boldsymbol{B}$ ) spatial frequency pattern of initial synaptic strengths. The evolution of synapses carrying information from the contralateral (top) and ipsilateral (bottom) eye, shown as color, versus time (horizontal) and position (vertical). C, The final spatial frequency (in cycles) of the ocular dominance columns, shown in color, as a function of the spatial frequency of the initial condition and the strength of the intracortical connections, $M_{A}$. The peak of the power spectrum of the function $M$, which describes the initial weight pattern when $M_{A}$ was not too large and when the spatial frequency of the initial weight pattern was three to six cycles close to the four-cycle spectrum peak of $M$; otherwise the frequency of the final $O D$ columns was set by the spectrum peak of $M$. (For better spatial resolution, $N=400$ neurons were simulated instead of $N=100$ in this panel.) The cortex did not equalize (the synaptic strength from one eye exceeded $60 \%$ of the total) within the simulation time when $M_{A}$ was too small (shown as white color). The cortex equalized before the maturation of inhibition when $M_{A}$ was too big (shown as black).
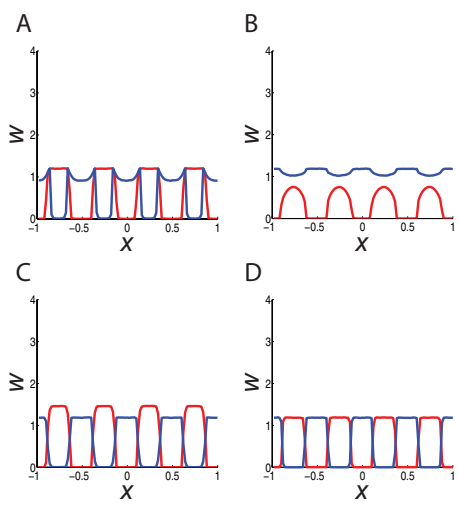

D

Figure 7. The synaptic strengths from the contralateral eye (red) and the ipsilateral eye (blue) after MD using the Hebbianplus-homosynaptic plasticity rule. The figure shows the results for different values of $f_{\mathrm{MD}}: 0(\boldsymbol{A}), 0.2(\boldsymbol{B}), 0.8(\boldsymbol{C})$, and $1(\boldsymbol{D})$. $\boldsymbol{E}$, The mean strength of the open-eye (ipsi) and closed-eye (contra) synapses after MD for differing values of $f_{M D}$ during MD.

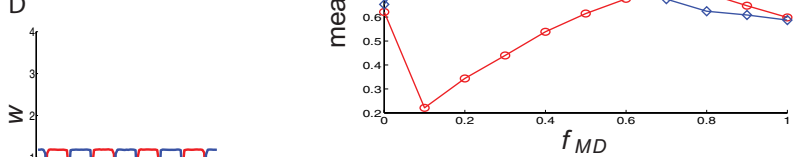

strengths and an increase of open-eye synapses as discussed above (Fig. $7 A, B)$ (as $f_{\mathrm{MD}}$ approaches zero, the MD effect almost disappears, because the rate of firing of the closed-eye inputs falls below the threshold for the LTD induced by weight decay, see Materials and Methods). However, if $f_{\mathrm{MD}}$ is larger than 0.7 , we observe an increase of closed-eye synaptic strengths (see Fig. 7C). In this case, the homeostatic rule leads to an increase in closedeye synapses rather than in open-eye synapses to compensate for the decreased postsynaptic activity. Although, the precise $f_{\mathrm{MD}}$ value for this switching behavior depends on parameters such as the weight decay coefficient $\gamma$ [making $\gamma$ smaller makes the 
closed-eye-potentiating domain larger (see supplemental material, available at www.jneurosci.org)], the qualitative feature seems to be robust. That is, if it were possible experimentally to induce a weak decrease in one eye's firing, one should see a homeostatic increase in that eye's synaptic strength, rather than the deprivation effect that occurs with a strong decrease in the eye's firing. This kind of partial decrease of the LGN activity corresponding to one eye might be achievable with a weakly blurring contact lens that reduces the effective contrast of stimuli seen through one eye. Finally, if $f_{\mathrm{MD}}=1$, as a control case, we see no significant changes in synaptic weights (see Fig. 7D).

\section{Discussion}

We have shown that the equalization of the input serving the two eyes that occurs at the onset of the critical period (CP) in cat V1, its failure to occur in the absence of visual experience (Crair et al., 1998), and the ability of monocular deprivation to drive ocular dominance shifts despite the tendency of cortex to equalize the two eyes' innervations, can all be understood from activitydependent rules of synaptic plasticity along with an experiencedependent maturation of inhibition at the onset of the CP like that observed in mice (Hensch et al., 1998; Fagiolini and Hensch, 2000). While the models used to demonstrate this are simple and imperfect, the underlying principles they expose are general and robust:

(1) Inhibition that is sufficiently strong will drive the innervations of the two eyes to equalize if they have comparable activities.

(2) To nonetheless obtain ocular dominance shifts in response to monocular deprivation, some mechanism must enable excitatory synapses driven by the open eye to grow under MD in closedeye-dominated regions, even when they cannot activate the postsynaptic cell, as is known to occur in vivo (Mioche and Singer, 1989). These synapses cannot activate the postsynaptic cell both because they are weak and because inhibition from open-eye-dominated regions is strong. Some combination of weakening of open-eye-driven inhibition and strengthening of open-eye-driven excitation must occur that brings the open eye to the point that it can successfully activate the postsynaptic cell.

We have shown that homeostatic plasticity of excitatory synapses, which in fact is implicated in the strengthening of open-eye synapses after MD (Kaneko et al., 2008b), along with a minimal amount of noise, is sufficient to achieve this. Alternatively, an LTP/LTD rule that favors LTP, along with strong noise and a rule such as weight normalization to force some synapses to shrink when others grow, can also achieve this.

Our model makes two clear predictions. First, just as in mice, a maturation of inhibition should occur in cat V1 to initiate the $\mathrm{CP}$, and visual deprivation (which prevents the equalization of the two eyes) should prevent this maturation. Second, just as early strengthening of inhibition, induced by infusion of benzodiazepine agonists (Fagiolini and Hensch, 2000; Iwai et al., 2003; Fagiolini et al., 2004) or excess brain-derived neurotrophic factor expression (Hanover et al., 1999; Huang et al., 1999), can cause an early initiation of the CP in mice, such treatment should cause early equalization of ocular dominance in cat V1. Given the slow onset of the effects of these treatments, this might most easily be observed by showing that the treatment rescues the delayed equalization of ocular dominance caused by visual deprivation.

The simplest rules of correlation-based plasticity with subtractive weight normalization can show equalization of ocular dominance in visual cortex, but the results are somewhat fragile: some parameter tuning is needed, and after inhibition leads to equalization, sufficient noise and a learning rule that is biased toward potentiation are needed for monocular deprivation to induce a loss of equalization. The fundamental problem with these simple rules is that they tend to push synaptic weights either to zero or to their maximal allowed values (Miller and MacKay, 1994), and they have no robust means by which weights that have been pushed to zero can recover after a change in input activity or in intracortical inhibition, although such recovery may occur for particular parameter choices. In reality, biology seems to have robust mechanisms by which even weights that have been driven to or near zero can bounce back under appropriate conditions (Mioche and Singer, 1989). The simplest learning rules do a reasonable job of modeling the emergence of a pattern from an initially unpatterned set of synapses, but do not have any robust mechanism for "unlearning" a pattern in which many synapses have zero or near-zero weights.

We have shown that one simple fix for these problems is found by adding a homeostatic learning mechanism (Turrigiano et al., 1998; Maffei et al., 2004; Turrigiano and Nelson, 2004; MrsicFlogel et al., 2007; Kaneko et al., 2008). While under stationary and homogeneous input, this homeostatic constraint is similar to the weight normalization of the total synaptic weight on a cell, it enables weights that are pushed to zero to recover strength if the postsynaptic cell is not well driven. We used a BCM-like rule (Bienenstock et al., 1982; Cooper et al., 2004) as a simple mechanism to instantiate this homeostasis, but this mechanism is not critical. In this rule, homeostasis arises because the threshold separating LTP from LTD changes faster than linearly with the mean postsynaptic activity. We have obtained similar results (data not shown) using a rule in which one term describes correlation-based LTP and LTD, without such a faster-thanlinear change in threshold, and a separate term causes synapses to be multiplicatively scaled in proportion to the deviation of average postsynaptic activity from a desired set point, as suggested by results on homeostatic synaptic scaling (Turrigiano et al., 1998; Maffei et al., 2004; Turrigiano and Nelson, 2004; Mrsic-Flogel et al., 2007; Kaneko et al., 2008). Indeed, an empirical problem with the BCM-like mechanism is that it enforces homeostasis through a subtractive rather than multiplicative change in synaptic strengths [note, however, homeostatic plasticity in developing visual cortex can also cause an additive change in neuronal responses by additively modifying the curve of firing rate vs input current (Maffei and Turrigiano, 2008)]. Multiplicative scaling shows all the same behaviors as the subtractive rule studied here (data not shown), so long as the minimal weight is above zero (so that synapses do not reach zero, from which they cannot recover under multiplicative scaling) and the LTP/LTD rule produces LTD of closed-eye synapses under MD.

We modified the BCM-like rule by adding a weight decay term (Eq. 7). This was necessary to explain the observation that, after $\mathrm{MD}$, deprived-eye synapses weaken before open-eye synapses strengthen (Mioche and Singer, 1989; Frenkel and Bear, 2004; Mrsic-Flogel et al., 2007; Kaneko et al., 2008). This cannot be captured by the BCM-like rule alone, because the homeostatic weight regulation and the correlation-based LTP/LTD are in the same term. The LTP induced by the homeostatic response to the loss of postsynaptic activity thus prevents LTD due to deprivation-induced reduction of presynaptic activity (this problem might be fixed by making the LTP/LTD threshold change slowly, but then the BCM rule loses stability, leading to weight oscillations). This problem can also alternatively be solved (data not shown) using a model with separate LTP/LTD and homeostasis terms, as described above, a model we will more systematically study elsewhere.

Mice do not show equalization of the two eyes' innervations: 
the binocular segment of mouse V1 remains dominated by the contralateral eye after the opening of the CP (Gordon and Stryker, 1996). Why does the maturation of inhibition not cause equalization in the mouse? Mice, like other rodents (Van Hooser et al., 2005), do not have OD columns. We suspect the explanation for the lack of equalization is closely tied to the explanation for the lack of OD columns. In mathematical and computational models, OD columns arise through a combination of two factors: cooperative interactions between nearby V1 cells that lead them to tend to receive correlated inputs, and more widespread competitive interactions that ensure that not all cells become dominated by the same inputs (Miller, 1990, 1996). In Hebbian models, the cooperative interactions typically involve excitatory connections between nearby cells. In this framework, the most likely explanation for the lack of columnar organization in rodents is that the effective connectivity between nearby neurons during development is either too weak to organize columns, or is of the wrong sign-net inhibitory rather than excitatory. As we have seen, strong net inhibition between cells can act as a competitive factor leading to equalization of inputs, so the lack of equalization may indicate that overall interactions are relatively weak during development. Note that inhibition onto each cell can be strong, as presumably occurs after the maturation of inhibition that initiates the $\mathrm{CP}$, without necessarily involving strong inhibitory effective interactions between excitatory cells, for example if inhibition is predominantly driven by feedforward rather than recurrent projections.

Mitchell et al. (1977) pointed out a similar problem of equalization: if a transition from MD to normal binocular experience was made sufficiently early, the originally deprived eye showed a significant, though not full, recovery both physiologically and behaviorally. That is, the weaker eye gained while both eyes had equal activity. They suggested that this might involve an advantage of the deprived eye in competing to reinnervate territory it lost during MD. This recovery process is now known to require BDNF (brain-derived neurotrophic factor) signaling, whereas the processes of MD do not (Kaneko et al., 2008). The equalization we are studying may involve a different mechanism, one test of which would be to determine whether it also requires BDNF.

Swindale (1980) modeled the data of Mitchell et al. (1977) as dynamic equalization under linear dynamics. He assumed a phenomenological interaction between synapses that depended on their eyes of origin and was a DOG function of their separation in cortex. The integral of the DOG determined the growth rate of the "DC" pattern, in which one eye dominates everywhere. He assumed the integral was negative, so that the DC pattern decayed to zero, yielding ocular equalization. MD added a "source" term to the dynamics of the DC pattern, causing the more active eye to dominate.

Our explanations of equalization and the ability to achieve MD effects despite equalization, although similar in spirit to Swindale's, differ from his in four respects, as discussed in more detail in supplemental material (available at www.jneurosci.org): (1) the spatial dependence of Swindale's phenomenological interaction has been identified, in the limit of spatially broad correlations, with the intracortical interaction function (Miller et al., 1989); (2) with this identification, the growth rate of the DC pattern is always positive (the growth rate is $\tilde{K}(0)=(1-$ $\tilde{M}(0))^{-1}$, where $\tilde{M}(0)<1$ and is proportional to the integral of the DOG describing intracortical connectivity); (3) nonlinear effects, whereby the faster growth of periodic patterns leads to suppression of the DC pattern, therefore are essential to equalization; and (4) to attain MD along with equalization becomes problematic in this scenario, and so requires specific solutions such as homeostatic plasticity to allow weak synapses to recover and compete, as we address here.

The idea that intracortical connectivity can create a periodic organization of receptive field properties and determine its period is an old one (van der Malsburg, 1973; Amari, 1977; Swindale, 1980; Kohonen, 1982; Miller et al., 1989; Castellani et al., 1999; Ernst et al., 2001; Koulakov and Chklovskii, 2001) Consistent with this idea, genetic factors, which might act by influencing the structure of intracortical connectivity, contribute to the spacing of cortical orientation and ocular dominance maps (Kaschube et al., 2002, 2003). A recent experiment on cats also demonstrated that a chronic increase in the strength of intracortical inhibition from large basket cells, which project horizontal axons at longer distances than other inhibitory cell types, widens the spacing of ocular dominance columns (Hensch and Stryker, 2004). As those authors noted, this is the expected result from simple models like those studied here if homeostatic mechanisms preserved the overall strength of inhibition after its maturation at the onset of the $\mathrm{CP}$, so that the overall effect of the chronic change was to relatively increase the strength of longer-range inhibition versus shorter-range inhibition. In contrast, the overall increase in the strength of inhibition that occurs at the onset of the CP would not be expected to widen column width.

The question of whether ocular dominance columns are genetically specified or arise through activity-dependent selforganization has been a controversial one in recent years (Crair et al., 2001; Crowley and Katz, 2002; Kaschube et al., 2002, 2003; Huberman, 2007) (see also Miller et al., 1999 on the same question with respect to orientation columns). In cats, activityindependent genetic factors alone could at most account for the condition of ipsilateral "islands" in a "sea" of contralateral inputs observed both physiologically and anatomically in the third postnatal week (Crair et al., 1998, 2001), because the subsequent equalization of inputs serving the two eyes and their organization into ocular dominance columns does not occur without visual experience (Crair et al., 1998). There has been no proposal as to how this experience-dependent equalization and reorganization could occur, and it has been repeatedly been argued that it could not be explained by activity-dependent self-organization (Crair et al., 1998; Crowley and Katz, 1999; Katz and Crowley, 2002). Here, we have shown that activity-dependent learning rules, together with a pattern of intracortical interactions that yields ocular dominance columns, provide a simple, robust, and testable explanation.

In summary, we argue that the observation in cat V1 that ocular dominance equalizes at the onset of the critical period may simply be a different window on the observation, made in mouse $\mathrm{V} 1$, that the critical period is initiated by the maturation of intracortical inhibition.

\section{References}

Abbott LF, Nelson SB (2000) Synaptic plasticity: taming the beast. Nat Neurosci 3 [Suppl]:1178-1183.

Amari S (1977) Dynamics of pattern formation in lateral-inhibition type neural fields. Biol Cybern 27:77-87.

Bienenstock EL, Cooper LN, Munro PW (1982) Theory for the development of neuron selectivity: orientation specificity and binocular interaction in visual cortex. J Neurosci 2:32-48.

Butts DA, Kanold PO, Shatz CJ (2007) A burst-based “Hebbian” learning rule at retinogeniculate synapses links retinal waves to activity-dependent refinement. PLoS Biol 5:e61.

Castellani GC, Intrator N, Shouval H, Cooper LN (1999) Solutions of the BCM learning rule in a network of lateral interacting linear neurons. Network 10:111-121.

Chiu C, Weliky M (2002) Relationship of correlated spontaneous activity to 
functional ocular dominance columns in the developing visual cortex. Neuron 35:1123-1134.

Cooper LN, Blais BS, Shouval HZ, Intrator N (2004) Theory of cortical plasticity. Singapore: World Scientific.

Crair MC, Gillespie DC, Stryker MP (1998) The role of visual experience in the development of columns in cat visual cortex. Science 279:566-570.

Crair MC, Horton JC, Antonini A, Stryker MP (2001) Emergence of ocular dominance columns in cat visual cortex by 2 weeks of age. J Comp Neurol 430:235-249.

Crowley JC, Katz LC (1999) Development of ocular dominance columns in the absence of retinal input. Nat Neurosci 2:1125-1130.

Crowley JC, Katz LC (2002) Ocular dominance development revisited. Curr Opin Neurobiol 12:104-109.

Ernst UA, Pawelzik KR, Sahar-Pikielny C, Tsodyks MV (2001) Intracortical origin of visual maps. Nat Neurosci 4:431-436.

Erwin E, Miller KD (1998) Correlation-based development of ocularlymatched orientation maps and ocular dominance maps: determination of required input activity structures. J Neurosci 18:9870-9895.

Fagiolini M, Hensch TK (2000) Inhibitory threshold for critical-period activation in primary visual cortex. Nature 404:183-186.

Fagiolini M, Fritschy JM, Löw K, Möhler H, Rudolph U, Hensch TK (2004) Specific GABAA circuits for visual cortical plasticity. Science 303:1681-1683.

Frenkel MY, Bear MF (2004) How monocular deprivation shifts ocular dominance in visual cortex of young mice. Neuron 44:917-923.

Gerstner W, Kistler WK (2002) Spiking neuron models. Cambridge, UK: Cambridge UP.

Gordon JA, Stryker MP (1996) Experience-dependent plasticity of binocular responses in the primary visual cortex of the mouse. J Neurosci 16:3274-3286.

Haider B, Duque A, Hasenstaub AR, McCormick DA (2006) Neocortical network activity in vivo is generated through a dynamic balance of excitation and inhibition. J Neurosci 26:4535-4545.

Hanover JL, Huang ZJ, Tonegawa S, Stryker MP (1999) Brain-derived neurotrophic factor overexpression induces precocious critical period in mouse visual cortex. J Neurosci 19:RC40.

Hensch TK, Stryker MP (2004) Columnar architecture sculpted by GABA circuits in developing cat visual cortex. Science 303:1678-1681.

Hensch TK, Fagiolini M, Mataga N, Stryker MP, Baekkeskov S, Kash SF (1998) Local GABA circuit control of experience-dependent plasticity in developing visual cortex. Science 282:1504-1508.

Heynen AJ, Yoon BJ, Liu CH, Chung HJ, Huganir RL, Bear MF (2003) Molecular mechanism for loss of visual cortical responsiveness following brief monocular deprivation. Nat Neurosci 6:854-862.

Higley MJ, Contreras D (2006) Balanced excitation and inhibition determine spike timing during frequency adaptation. J Neurosci 26:448-457.

Huang ZJ, Kirkwood A, Pizzorusso T, Porciatti V, Morales B, Bear MF, Maffei L, Tonegawa S (1999) BDNF regulates the maturation of inhibition and the critical period of plasticity in mouse visual cortex. Cell 98:739-755.

Huberman AD (2007) Mechanisms of eye-specific visual circuit development. Curr Opin Neurobiol 17:73-80.

Iwai Y, Fagiolini M, Obata K, Hensch TK (2003) Rapid critical period induction by tonic inhibition in visual cortex. J Neurosci 23:6695-6702.

Kaneko M, Stellwagen D, Malenka RC, Stryker MP (2008) Tumor necrosis factor-alpha mediates one component of competitive, experiencedependent plasticity in developing visual cortex. Neuron 58:673-680.

Kang K, Shelley M, Sompolinsky H (2003) Mexican hats and pinwheels in visual cortex. Proc Natl Acad Sci U S A 100:2848-2853.

Kaschube M, Wolf F, Geisel T, Löwel S (2002) Genetic influence on quantitative features of neocortical architecture. J Neurosci 22:7206-7217.

Kaschube M, Wolf F, Puhlmann M, Rathjen S, Schmidt KF, Geisel T, Löwel S (2003) L columns in cat primary visual cortex: intra- and interindividual variability of column spacing and its dependence on genetic background. Eur J Neurosci 18:3251-3266.

Katz LC, Crowley JC (2002) Development of cortical circuits: lessons from ocular dominance columns. Nat Rev Neurosci 3:34-42.

Katz LC, Shatz CJ (1996) Synaptic activity and the construction of cortical circuits. Science 274:1133-1138.

Kohonen T (1982) Self-organized formation of topologically correct feature maps. Biol Cybern 43:59-69.

Koulakov AA, Chklovskii DB (2001) Orientation preference patterns in mammalian visual cortex: a wire length minimization approach. Neuron 29:519-527.

Linden ML, Heynen AJ, Haslinger RH, Bear MF (2009) Thalamic activity that drives visual cortical plasticity. Nat Neurosci 4:390-392.

Lisman J, Spruston N (2005) Postsynaptic depolarization requirements for LTP and LTD: a critique of spike timing-dependent plasticity. Nat Neurosci 8:839-841.

Maffei A, Turrigiano GG (2008) Multiple modes of network homeostasis in visual cortical layer 2/3. J Neurosci 28:4377-4384.

Maffei A, Nelson SB, Turrigiano GG (2004) Selective reconfiguration of layer 4 visual cortical circuitry by visual deprivation. Nat Neurosci 7:1353-1359.

Miller KD (1990) Correlation-based models of neural development. In: Neuroscience and connectionist theory (Gluck MA, Rumelhart DE, eds), pp 267-353. Hillsdale, NJ: Erlbaum.

Miller KD (1996) Receptive fields and maps in the visual cortex: models of ocular dominance and orientation columns. In: Models of neural networks III (Domany E, van Hemmen JL, Schulten K, eds), pp 55-78. New York: Springer.

Miller KD, MacKay DJC (1994) The role of constraints in Hebbian learning. Neural Comput 6:100-126.

Miller KD, Keller JB, Stryker MP (1989) Ocular dominance column development: analysis and simulation. Science 245:605-615.

Miller KD, Erwin E, Kayser A (1999) Is the development of orientation selectivity instructed by activity? J Neurobiol 41:44-57.

Mioche L, Singer W (1989) Chronic recordings from single sites of kitten striate cortex during experience-dependent modifications of receptivefield properties. J Neurophysiol 62:185-197.

Mitchell DE, Cynader M, Movshon JA (1977) Recovery from the effects of monocular deprivation in kittens. J Comp Neurol 176:53-63.

Morales B, Choi SY, Kirkwood A (2002) Dark rearing alters the development of GABAergic transmission in visual cortex. J Neurosci 22:8084-8090.

Mrsic-Flogel TD, Hofer SB, Ohki K, Reid RC, Bonhoeffer T, Hübener M (2007) responses in visual cortex during ocular dominance plasticity. Neuron 54:961-972.

Pinto DJ, Ermentrout GB (2001) Spatially structured activity in synaptically coupled neuronal networks: II. Lateral inhibition and standing pulses. SIAM J Appl Math 62:226-243.

Rittenhouse CD, Shouval HZ, Paradiso MA, Bear MF (1999) Monocular deprivation induces homosynaptic long-term depression in visual cortex. Nature 397:347-350.

Shu Y, Hasenstaub A, McCormick DA (2003) Turning on and off recurrent balanced cortical activity. Nature 423:288-293.

Song S, Miller KD, Abbott LF (2000) Competitive Hebbian learning through spike-timing-dependent synaptic plasticity. Nat Neurosci 3:919-926.

Stryker MP (1986) The role of neural activity in rearranging connections in the central visual system. In: The biology of change in otolaryngology (Ruben RJ, Van De Water TR, Rubel EW, eds), pp. 211-224. Amsterdam: Elsevier Science.

Swindale NV (1980) A model for the formation of ocular dominance stripes. Proc R Soc Lond B Biol Sci 208:243-264.

Swindale NV (1996) The development of topography in the visual cortex: a review of models. Network 7:161-247.

Turrigiano GG, Nelson SB (2004) Homeostatic plasticity in the developing nervous system. Nat Rev Neurosci 5:97-107.

Turrigiano GG, Leslie KR, Desai NS, Rutherford LC, Nelson SB (1998) Activity-dependent scaling of quantal amplitude in neocortical neurons. Nature 391:892-896.

Van Hooser SD, Heimel JA, Chung S, Nelson SB, Toth LJ (2005) Orientation selectivity without orientation maps in visual cortex of a highly visual mammal. J Neurosci 25:19-28.

van Vreeswijk C, Sompolinsky H (1998) Chaotic balanced state in a model of cortical circuits. Neural Comput 10:1321-1371.

von der Malsburg C (1973) Self-organization of orientation selective cells in the striate cortex. Kybernetik 14:85-100.

Wehr M, Zador AM (2003) Balanced inhibition underlies tuning and sharpens spike timing in auditory cortex. Nature 426:442-446.

Weliky M, Katz LC (1998) Correlational structure of spontaneous activity in the developing lateral geniculate nucleus in vivo. Science 285:599-604. 